\title{
NEUROPHYSIOLOGICAL EVALUATION OF UNDERGRADUATE PORTUGUESE YOUNG ADULTS WITH READING DIFFICULTIES
}

\author{
Marta Alves, Patrícia Pinto, Diana Tavares, \& Ana Santos \\ Escola Superior de Saúde, Instituto Politécnico do Porto (Portugal)
}

\begin{abstract}
Introduction: Reading is a complex cognitive process that requires a simultaneous activation of different brain systems. Power EEG has been used to study activation patterns in dyslexic subjects, but so far, studies lack result's coherence, including in adult dyslexic population. The Reading Age Test (TIL) is a screening assessment for dyslexia. TIL evaluates the processes of decoding and understanding. This test is adapted and validated to Portuguese language. Objective: To analyze neurophysiological differences in undergraduate adults with and without reading difficulties using power EEG, and compare the results with the ones found at younger ages. Methods: 209 college students were administered the TIL. EEG was collected with 26 students ( 21 within normal reading level; 5 with severe reading difficulties signalized with TIL). During the EEG, the participant was asked to follow a sequence of tasks, during a total of 15 minutes (Basal resting state, TIL, Pos-TIL resting state, Non-reading Task; final resting state). Subsequently, the Fourier Transform (FFT) algorithm was applied to the EEG signal from the Basal resting state and the two given tasks. Power spectra mean values of delta, theta and beta activity band, from electrodes F7, F8, T3, T4, T5 and T6 were analyzed performing Shapiro-Wilk tests. Results: No significant differences in mean variations $(\mathrm{sig}>0.05)$ were observed between groups during the analyzed periods, regarding electrode and brain activity band frequency. Conclusion: This study provided inconclusive results concerning power EEG different findings at the lower frontal gyrus region and temporal region, between subject with and without reading difficulties.
\end{abstract}

Keywords: Dyslexic students, TIL, power EEG.

\section{Introduction}

Reading is a complex cognitive process, composed of actions of apprehension and understanding, which requires the simultaneous activation of several neurological systems, namely the visual, auditory, motor, and attention. The existence of any change or deficit in one of these systems will affect the functioning of the others, leading to difficulties that can manifest in imprecise and often slow decoding of the written language, resulting in inefficient reading. Dyslexia presents itself as a heterogeneous phenotype syndrome, with a pronounced and persistent difficulty in the acquisition of reading, despite normal intelligence, sensory acuity, motivation and educational. (Snowling \& Hulme, 2011; Spironelli, Penolazzi, \& Angrilli, 2008).

The reading activity is based on the left hemisphere, and implicates three circuits. These are located in the occipito-temporal region, responsible for visual processing and word recognition, in the temporo-parietal region, which encompasses the superior, supra-marginal and angular temporal gyres, responsible for phonological, orthographic and semantic processing and for grapheme-phoneme conversion, and in the region of the lower frontal gyrus, involved in phonological processing and speech articulation. It is suggested that dyslexia is related to structural changes in neuronal systems, i.e. specific cortical changes in the left peri-silvian region, as well as inter-hemispheric asymmetries, which focus on the temporal planum. In diagnosed individuals, there's record of decreased activation of neuronal circuits in the temporo-parietal and occipito-temporal regions, followed by an increased activation in the inferior frontal region, during the performing of reading related tasks. (Evans \& Park, 2005; Leonard \& Eckert, 2009; Penolazzi, Spironelli, Vio, \& Angrilli, 2010).

EEG is a method of easy execution, relatively innocuous and affordable, which allows a post recorded analysis of brain activity. The power EEG is the result of the application of the Fourier Transform (FFT) algorithm to the conventional EEG signal, allowing to quantify the brain activity registered in the scalp.(Kaiser, Lennert, \& Lutzenberger, 2007; Kuba et al., 2001; Teixeira, 2015; Thornton \& Carmody, 2012). Mental processes cause oscillations in the EEG that have to be taken into account for their interpretation. The thalamus-reticular nucleus inhibits the random firing of thalamocortical neurons and gadders these individual discharges, making them into one larger discharge, which spreads to the cortex, synchronizing the activity of the pyramidal cells. This synchronization can be detected in the EEG through wide variations. There is then a corticotalamic feedback that influences the 
inhibition of the thalamus-reticular nucleus. When information processing occurs, this feedback will inhibit the inhibition of the thalamus-reticular nucleus itself, which leads to brain activity free of reticule-thalamic influence, thus being able to respond to the processing. This process is titled EEG desynchronization.(Kaiser et al., 2007). The EEG desynchronization of an area reflects neuronal groups that work independently, with maximum responsiveness and information, meaning an increase in excitability of the cortex. EEG synchronization reflects neuronal groups that work simultaneously, mirroring reduced information processing, and decrease in cortical excitability.(Kaiser et al., 2007; Lemm, Müller, \& Curio, 2009).

The majority of studies regarding analysis of EEG in dyslexic subjects leans over younger subjects and show a variance of results. Some studies report an increasing in spectral power of delta activity, associated with a slight increasing in spectral power of theta and activity, in frontal, central and temporal regions in dyslexic individuals. Was also observed an increasing in spectral power of beta activity in the left occipital region. In other hand, there were cases where no conclusive differences were observed between dyslexic subjects and normal readers.(Arns, Peters, Breteler, \& Verhoeven, 2007; Duffy, Denckla, Bartels, \& Sandini, 1980; Evans \& Park, 2005; Fein et al., 1986).

The present research intended to analyze neurophysiological differences regarding power EEG between undergraduate young adults with reading difficulties and a normal reading level, by exploring brain activity at a resting state, during the performance of a reading task and during the performance of a non-reading task. The following electrodes, F7, F8, T3, T4, T5 and T6, were considered as a starting point in the study, and delta, theta and beta frequency brain activity bands were evaluated.

\section{Methods}

\subsection{Reading Age Test (TIL)}

The Reading Age Test (TIL) is a screening test for reading difficulties, evaluating the processes of decoding and understanding, two essential skills in the task of reading. This test, easy to apply and with the possibility of being administered in a group, adapted and validated to the Portuguese language, may be adopted as step one in dyslexia diagnosis. It consists of 36 sentences. The subject reads the sentences in silence and completes them, by choosing the correct word out of five given options. The college student's version takes 2,5 minutes to be completed. The final score is achieved by the formula: Final grade $=$ (number of sentences completed correctly $\mathrm{x} 100) / 36$. The score is then compared to population's reference values.

The test was applied during classes. Instructions were given by the investigator out loud, and all students started the test at the same moment, having two minutes and thirty seconds to complete it. After scoring, subjects with a twenty-five or below correct answers (subjects with reading difficulties, at or below the 5th percentile) and twenty-seven or above correct answers (subjects with normal reading level) were contacted by the investigators.

\subsection{Non-reading task}

The non-reading task (NRT) required the subject to order four pictures that mimic a daily life action, repeating the same task six times with six different groups of pictures, having fifteen seconds to each group. This was used as control task.

\subsection{Participants}

The study was conducted in the facilities of Escola Superior de Saúde do Porto. The first phase included volunteer bachelor's students, 18 years or more, and Portuguese language as mother language. In total, 209 subjects (169 females; 40 males; mean age: $20.21 \pm 4.11)$ met the criteria and agreed to take the test. After scoring, subjects with a 25 or below correct answers $(\mathrm{N}=85)$ and 27 or above correct answers $(\mathrm{N}=106)$ were contacted by the investigators, in order to continue the study. To continue the study, subjects could not meet any exclusion criteria: mental illness and neurological disorder in person/ familiar history, uncorrected visual/auditory deficit, and use psychotropic drugs. 31 subjects (25 female; 6 male) contacted the investigators. Two subjects met at least two exclusion criteria points, and were excluded. From the twenty-nine EEG performed, three were excluded because of signal's collection failure during the exam that compromised the results. The subjects were divided in two groups: Group A ( 2 female; 3 male; age mean: $19.80 \pm 1.09$; second TIL score $\leq 23$ correct answers or 5 th percentile) and Group B (17 female; 4 male; age mean: $20.29 \pm 1.74$; second TIL score $\leq 31$ correct answers or 60 th percentile).

\subsection{EEG: Data acquisition and analysis}

The EEG data were acquired the Deymed equipment - Software Truescan Acquision, using the internationally System 10-20-electrode placement, Fpz as reference and FCz as ground. The impedance was kept bellow $10 \mathrm{kOhms}$. The acquisition took place in a room with controlled sound and minimal light. The EEG had a total duration of 15 minutes, and was compose by the followed sequence: 3 minutes 
of recording basal activity (alternating eyes close/open every minute); performance of TIL during a maximum of 2,5 minutes and resting period with closing eyes; performance of NRT during 1,5 minutes; final resting period with closing eyes. All subjects were instructed to not talk during the EEG and to avoid sudden or repetitive movements.

From each EEG were analyzed 3 periods with 10 second length each in the beginning, middle and end of the basal, the TIL performance and the NRT period. The selected periods were the same time wise in all subjects, to the exception of presence of artefact that could compromised the results. The EEG signal selected was converted into power spectra. Investigators analyzed power spectra mean values of delta, theta and beta activity band, from electrodes F7, F8, T3, T4, T5 and T6 in the mentioned periods. Mean variations were analyzed performing Shapiro-Wilk tests. Mann-Whitney tests were applied in order to order compare the results between the two groups and different periods. The evolution of the subject's TIL score and the non-reading task score between groups was also analyzed.

\section{Results}

There were no observed significant differences in mean variations $(p>0.05)$ between Group A and Group B during resting state period, TIL performance period and Non-reading task period, regarding electrode and brain activity band frequency. Concerning the TIL score, all participants increased their score during the test taken upon the EEG data collection. However, participants with reading difficulties were not able to reach a score above the 5th percentile. The Non- reading task's results didn't show a direct correlation between reading and non-reading performance, showing no significant mean variations.

\section{Conclusions}

This study focused on neurophysiological characteristics concerning power EEG different findings at the lower frontal gyrus region and temporal region, between a group with reading difficulties and a group with normal reading level, in undergraduate students. Results were inconclusive, as there were no significant difference in mean variations between the two groups.

The EEG can be an error-prone method if the presence of artifacts is not taken into account, and the use of artefact removal algorithms can be seen as a vantage. In future studies, it is suggested the analysis of brain activity's amplitude variations. It is also recommended to increase the sample of participants with reading difficulties.

\section{References}

Arns, M., Peters, S., Breteler, R., \& Verhoeven, L. (2007). Different Brain Activation Patterns in Dyslexic Children: Evidence from Eeg Power and Coherence Patterns for the Double-Deficit Theory of Dyslexia. Journal of Integrative Neuroscience, 06(01), 175-190.

Duffy, F. H., Denckla, M. B., Bartels, P. H., \& Sandini, G. (1980). Dyslexia: Regional differences in brain electrical activity by topographic mapping. Annals of Neurology, 7(5), 412-420.

Evans, J. R., \& Park, N.-S. (2005). Quantitative EEG Abnormalities in a Sample of Dyslexic Persons. Journal of Neurotherapy, 2(1), 1-5.

Fein, G., Galin, D., Yingling, C. D., Johnstone, J., Davenport, L., \& Herron, J. (1986). EEG spectra in dyslexic and control boys during resting conditions. Electroencephalography and Clinical Neurophysiology, 63(2), 87-97.

Kaiser, J., Lennert, T., \& Lutzenberger, W. (2007). Dynamics of oscillatory activity during auditory decision making. Cerebral Cortex, 17(10), 2258-2267.

Kuba, M., Szanyi, J., Gayer, D., Kremlacek, J., Kubova, Z., Kremlácek, J., \& Kubová, Z. (2001). Electrophysiological testing of dyslexia. Acta Medica, 44(4), 131-134. Retrieved from

Lemm, S., Müller, K. R., \& Curio, G. (2009). A generalized framework for quantifying the dynamics of EEG event-related desynchronization. PLoS Computational Biology, 5(8).

Leonard, C. M., \& Eckert, M. A. (2009). Asymmetry and Dislexia. Brain, 33(6), 663-681.

Penolazzi, B., Spironelli, C., Vio, C., \& Angrilli, A. (2010). Brain plasticity in developmental dyslexia after phonological treatment: A beta EEG band study. Behavioural Brain Research, 209(1), 179-182.

Snowling, M. J., \& Hulme, C. (2011). Annual Research Review: The nature and classification of reading disorders: a commentary on proposals for DSM-5.

Spironelli, C., Penolazzi, B., \& Angrilli, A. (2008). Dysfunctional hemispheric asymmetry of theta and beta EEG activity during linguistic tasks in developmental dyslexia. Biological Psychology, 77(2), 123-131.

Teixeira, J. M. (2015). qEEG : Sua utilidade na prática clínica psiquiátrica. Revista Portuguesa de Psiquiatria e Saúde Mental, 1(1), 11-20.

Thornton, K. E., \& Carmody, D. P. (2012). Symbol Digit and the Quantitative EEG. Journal of Neurotherapy, 16(3), 210-222. 